Derek Richards UK Associate Editor

\section{Selection Criteria for Dental Radiography}

Faculty of General Dental Practitioners (UK). The Royal College of Surgeons of England.

Selection

Criteria

for Dental

Radiography

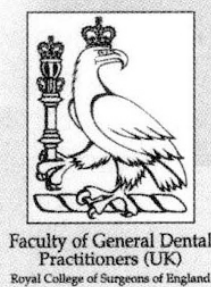

Royal College of Surgenns of Bnglant

This very useful ring bound A5 book was produced by an expert panel assembled by the Faculty of General Dental Practitioners (UK). Professor Nigel Pitts chaired the panel and the whole process facilitated by the Dental Health Services Research Unit at Dundee.

In compiling the book the panel has followed the Scottish Intercollegiate Guidelines Network (SIGN) methodol$\mathrm{ogy}^{1}$ and this is outlined in appendix A of the book.

The result is a clearly laid out book in eight sections covering:

1. Use of ionising radiation

1.1. Radiation dose and risks in dental practice

1.2.The use of panoramic radiography

2. Radiographs in the management of the developing dentition
3. Radiographs in dental caries diagnosis

4. Radiographs in periodontal assessment

5. Radiographs in the heavily restored dentition

6. Radiographs in endodontics

7. Radiographs in implantology

8. Good Practice

In each section there a summary statements and guidelines. The levels of evidence supporting these statements are clearly indicated using an easily understood grading system consistent with the SIGN system of classification. The book has a number of foldout pages summarising various elements of the text. There is also a date for revisiting and revising the guideline which is an important element of the SIGN approach. Let us hope that this target is met because new research evidence is constantly being produced.

This book is a substantial achievement and the Faculty and the expert panels are to be congratulated on what is probably the first evidence-based guideline available in dentistry. The most disappointing aspect of the guideline is that the number of statements that have the highest categories of evidence supporting them is small. One of the aims of this text is to improve standards of radiographic decision making in dentistry. I sincerely hope that it is instrumental in achieving this. Let us also hope that having identified both further areas of research and other areas where the available research is not of the highest quality that this may go some way to improving it.

1 http:/pc47.cee.hw.ac.uk/sign 